\title{
COLLOIDAL PROCESSING AND SINTERING OF NANOSIZED TRANSITION ALUMINAS
}

\author{
Paul Bowen ${ }^{1 *}$, Claude Carry ${ }^{2}$, David Luxembourg ${ }^{1}$, Heinrich Hofmann $^{1}$ \\ 1-Laboratoire de Technologie des Poudres (LTP), Département des Matériaux, Ecole \\ Polytechnique Fédérale de Lausanne (EPFL), CH 1015 Lausanne, Suisse. \\ *paul.bowen@epfl.ch \\ 2-Laboratoire de Thermodynamique et de Physico-Chimie Métallurgiques (LTPCM), UMR 5614 \\ CNRS-INPG/UJF, ENSEEG B.P. 75 Domaine Universitaire, 38402 SAINT MARTIN D'HERES \\ Cedex, France
}

\begin{abstract}
The dispersion of nanosized gamma aluminas with high specific surfaces areas $\left(100 \mathrm{~m}^{2} / \mathrm{g}\right)$ and primary particle sizes around $20 \mathrm{~nm}$, using polyacrylic acid has been investigated. The effect of $\mathrm{pH}$ and polymer concentration showed that the highest density green bodies were produced using high polymer concentrations $(6 \%$ wt) and $\mathrm{pH}$ of 6 . Interparticle potential calculations have been made and help explain the underlying dispersion mechanism at least on a qualitative level. The dispersions were then used to slip cast green bodies followed by drying and sintering. The types of gamma alumina powder have been investigated, the pure gamma alumina, doped with $\mathrm{MgO}$ and also with the addition of alpha alumina seeds. The high degree of agglomeration of the gamma alumina powders led to very low densities $(60 \%)$ even the alpha seeded alumina reached only $85 \%$ theoretical density. Attrition milling with zirconia media improves both green density and sintered densities significantly with all powders showing sintered densities $>97 \%$. Mictrostructural analysis on polished and etched surfaces show, however, that the grain sizes are well above 1 micron over 50 times greater than the initial gamma alumina primary particles. A two step sintering cycle was investigated with the $\mathrm{Mg}$ doped powder and average grain sizes around 580 nm were achieved.
\end{abstract}

\section{INTRODUCTION}

The quest to produce fully dense nanostructured ceramics has received much attention over the recent years because of the promise of nanograined materials with new and enhanced properties [1]. One of the main difficulties in realizing the promise of new nanograined ceramics has been in the difficulty of processing nanosized powders and producing fully dense components. When trying to achieve full density, grain growth out of the nanometre regime $(100 \mathrm{~nm})$ is often difficult to avoid [2] and microstructures are no finer than traditional sub-micron processed ceramics. Frequently, the starting nanopowder is not the thermodynamically stable phase at the processing temperature and there are phase transformations during the densification process. A typical example is the gamma alumina investigated in the present study [3]. Many attempts have been made to produce dense nanograined ceramics from transition aluminas, where both the phase transformations into the thermodynamically stable alpha alumina just above $1000^{\circ} \mathrm{C}$ and the thermal and processing history play important roles $[4,5]$.

Nanopowders are often made up of agglomerates 10-20 times larger than the primary nanoparticle. One major factor in the densification of nanopowder compacts is to stimulate interagglomerate densification and to avoid a predominance of intra-agglomerate densification, that leave large stable pores in the final piece. In this study we have investigated the properties of colloidal dispersions of a nanosized transition alumina $\left(\gamma-\mathrm{Al}_{2} \mathrm{O}_{3}\right)$ to improve the particle packing behaviour and reduce the tendency for intra-agglomerate sintering. We have also investigated 
seeding with alpha alumina that may influence both the phase transformation and sintering. Ceramic forming methods using colloidal suspensions can help improve green microstructures by minimising the number of agglomerates, increasing the packing uniformity, and lowering the average pore sizes [6]. To avoid agglomeration during slip casting most dispersions used are stabilised either by electrostatic repulsion or steric hindrance. A particularly efficient way of stabilising alumina suspensions is by the adsorption of a polyelectrolyte such as polyacrylic acid (PAA) which combines both repulsion mechanisms [7].

The current study is focused on the role of polyacrylic acid in the slip casting of gamma alumina suspensions. We have investigated two $\mathrm{pH}$ regimes ( $\mathrm{pH} 6$ and 10) where the modes of adsorption of the PAA molecule are expected to be quite different. At $\mathrm{pH} 6$ the surface of the alumina is positively charged and the PAA totally dissociated - leading to a "pancake" type of adsorbed layer [8]. At $\mathrm{pH} 10$ the alumina surface is negatively charged and although there are still adsorption sites available for the PAA, the conformation at the surface is expected to be more "brush"-like. This "brush"-like conformation and recent Atomic force microscopy (AFM) experiments suggest that the dominant mechanism of stabilisation is the steric contribution [9]. This leads us to the conclusion that the more extended conformation of the PAA at $\mathrm{pH} 10$ should give the most stable suspension. The amount of PAA adsorbed at the different pHs was investigated and adsorption isotherms collected. For the slip casting experiments the amount of PAA in the dispersion was varied from $1-6 \% \mathrm{wt}$ and the highest green densities were achieved with $6 \% \mathrm{wt}$ solutions at $\mathrm{pH} 6$ a somewhat unexpected result. The variation in zeta potential, green density, PAA adsorption isotherms and its conformation will be discussed in detail to help elucidate this somewhat unexpected behaviour.

On sintering even the best green bodies gave relatively poor sintered densities of around $80 \%$. This is mainly due to the state of agglomeration of the powder used [4] The sintered densities can be significantly improved by either attrition milling or seeding with alpha alumina and sintered densities over $98 \%$ can be achieved. Using either seeding or milling the grain sizes have still been above a micron. Sintering of gamma alumina milled and slip cast compacts using a two stage sintering regime, as used by Chen and Wang [10] to produce fully dense yttria ceramics, has also been investigated.

\section{MATERIALS AND METHODS}

The powders used were a high purity (99.995) $\gamma-\mathrm{Al}_{2} \mathrm{O}_{3}$ powder (type CR 125 Baikowski, France) with a specific surface areas of around $100 \mathrm{~m}^{2} / \mathrm{g}$. Three types of powder were investigated a pure gamma alumina, a magnesium doped gamma alumina $(\mathrm{Mg} / \mathrm{Al}$ ratio $6799 \mathrm{ppm})$ and a gamma alumina containing $9.4 \%$ alpha alumina seeds $\left(\mathrm{d}_{\mathrm{v} 50}\right.$ around $\left.250 \mathrm{~nm}\right)$. Particle size distributions (Table 1) were measured using an X-ray disc centrifuge (Bi-XDC, Brookhaven USA) with the density corrected for the porosity in the agglomerates estimated from nitrogen adsorptiondesorption isotherms (around $0.8 \mathrm{~cm}^{3} / \mathrm{g}$ ). Samples were slip cast in cylindrical rubber moulds $(10$ $\mathrm{mm}$ dia.) and height of around $10 \mathrm{~mm}$ after drying. The suspending medium for the slip preparation was a 1-6wt\% polyacrylic acid (PAA mol. wt. 2000) aqueous solution at pHs of 6 and 10 (adjusted with $\mathrm{NH}_{4} \mathrm{OH}$ ). Slurries were prepared from the as-received powders and after attrition milling for $3 \mathrm{hrs}$ (PE075 Netzsch, Germany). The milling media were $1.25 \mathrm{~mm}$ zirconia beads with $30 \mathrm{~g}$ powder, $70 \mathrm{~g}$ liquid and $700 \mathrm{~g}$ beads introduced into the attrition mill. The slip cast samples were dried in three stages, first at $90 \%$ relative humidity $(\mathrm{RH})$ followed by $45 \%$ 
(RH) and finally in a desiccator with silica-gel. The whole drying process took around 7 days. The dispersants were burnt out by heating in air at $1 \%$ min to $600^{\circ} \mathrm{C}$ and held for $1 \mathrm{hr}$. Samples were sintered using 2 routes; the first for screening and comparison, in air using heating rates of $10 \%$ min at $1600^{\circ} \mathrm{C}$ with no isothermal hold; the second to investigate the effect of a two step treatment similar to that proposed by Chen and Wang [10], heating at $10^{\circ} / \mathrm{min}$ to $1390^{\circ} \mathrm{C}$ reduced quickly to $1340^{\circ} \mathrm{C}$ and held for $20 \mathrm{hrs}$. Dilatometry and differential thermal analysis (Setaram, France) were carried out under similar conditions to the sintering cycles investigated. The microstructures of the sintered ceramics were observed on gold coated fracture surfaces and on polished and thermally etched surfaces by SEM (JEOL 6300, Japan). The thermal etching was carried out in $10 \% \mathrm{H}_{2}$ in Ar mixtures for 30 minutes at $150^{\circ} \mathrm{C}$ below the sintering temperature. Sample densities were measured using the Archimedes method. The percentage relative densities were calculated using 3.41 and $3.987 \mathrm{~g} / \mathrm{cm}^{3}$ for the gamma and alpha alumina phases respectively. The zirconia contamination from the milling process was measured using X-ray fluorescence spectroscopy and the zirconia content, generally around $2 \%$, was used to correct the calculated relative densities. The PAA isotherms were measured using $2 \mathrm{~g}$ of powder in $10 \mathrm{~g}$ of PAA solution at $25^{\circ} \mathrm{C}$ in an agitated bath for $24 \mathrm{hrs}$. The suspension was centrifuged and the supernatant analysed for the remaining PAA content by titration.

Table 1. Some powder characteristics for the aluminas studied.

\begin{tabular}{lccccc}
\hline Sample & $\% \alpha$ & $S S A\left(\mathrm{~m}^{2} / \mathrm{g}\right)$ & $d_{v 10}(\mathrm{~nm})$ & $d_{v 50}(\mathrm{~nm})$ & $d_{v 90}(\mathrm{~nm})$ \\
\hline CR 125 & $<0.3$ & 108 & $<111$ & 356 & 966 \\
Milled (PAA) & $<0.3$ & - & 30 & 52 & 151 \\
Mg Doped (Mg/A1 6799 ppm) & $<0.5$ & 94.8 & $<111$ & 381 & 1120 \\
Mg Doped milled & $<0.5$ & - & $<22$ & 49 & 103 \\
Seeded (9.4\% wt alpha) & 9.4 & 93.0 & 117 & 525 & 1201 \\
Seeded (9.4\% wt alpha) - milled & 9.4 & - & $<22$ & 54 & 205 \\
\hline
\end{tabular}

\section{RESULTS AND DISCUSSION}

\section{Colloidal stability of gamma alumina suspensions dispersed with PAA}

The amount of PAA adsorbed at the different $\mathrm{pH}$ 's was investigated and adsorption isotherms collected (Figure 1). At pH 10 a plateau in the adsorption isotherm seems to have been reached after the first adsorption point which corresponds to a $1 \% \mathrm{wt}$ PAA solution. The adsorbed amount at this plateau is around $27 \mathrm{mg} \mathrm{PAA} / \mathrm{g}$ powder. In contrast at $\mathrm{pH} 6$ the amount adsorbed is about double at around $52 \mathrm{mg} \mathrm{PAA} / \mathrm{g}$ powder and increases steadily as the PAA solution concentration increases.

The zeta potential of the gamma alumina suspensions (in $0.01 \mathrm{M} \mathrm{KNO}_{3}$ ) as a function of $\mathrm{pH}$ is shown in Figure 2(a) with the expected form and isoelectric point ( $\mathrm{pH}$ 9.4) for alumina. The 
lower adsorbed amount of PAA at $\mathrm{pH} 10$, can be understood by considering that the majority of the surface is negatively charged and that the totally dissociated PAA is also negatively charged.

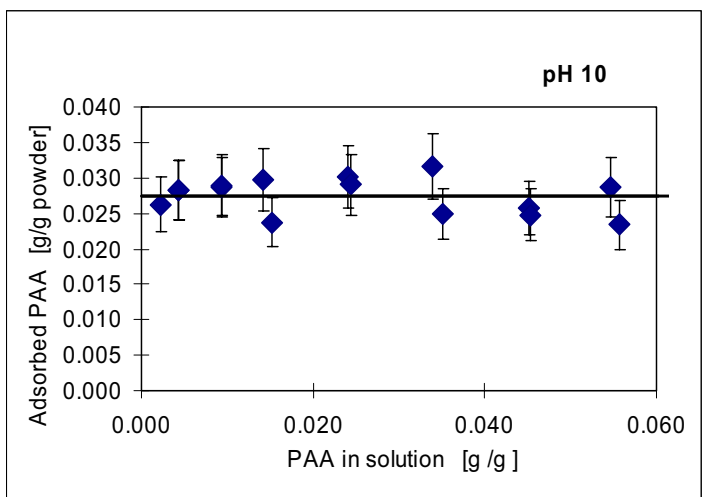

(a)

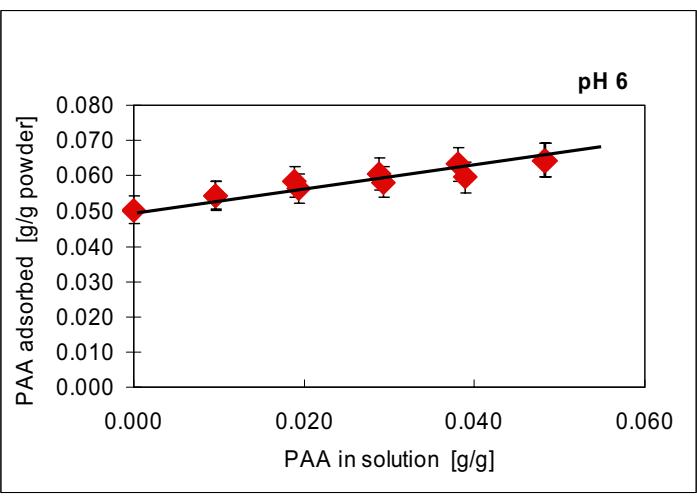

(b)

Figure 1. Adsorption isotherms of PAA onto gamma alumina at (a) pH 10 and (b) pH 6

Such a hypothesis results in a limitation of the number of favourable adsorption sites, which should lead to a "brush" like configuration as shown schematically in Figure 2 (c). Whereas at $\mathrm{pH} 6$ the surface is positively charged and the adsorption will be more of a "pancake" conformation (Figure 2 (b)). Although these conformations are probable from AFM measurements carried out on similar systems $[8,11]$ the adsorption conformations remain hypotheses. The surface coverage will depend on the conformation and space filling capacity of the PAA molecule. A random packing of the flat "pancake" conformation will lead to a certain surface coverage but because of steric hinderance and electrostatic repulsion between the PAA molecules and molecular segments it will not be a total surface coverage (e.g. even for monosized discs random close packing is around 54\%). When increasing the concentration of PAA in solution at $\mathrm{pH} 6$ the increased adsorption may be explained by molecules adsorbing in a more "brush like conformation as expected for the $\mathrm{pH} 10$ case where only small patches of positive surface sites are available (Figure 2(d)). These "brush" sites at $\mathrm{pH} 6$ are probably not that energetically favourable at low PAA concentrations (1\% wt PAA). Nevertheless, when the concentration in the solution increases, adsorption onto these sites is driven by the PAA excess in solution.

Slip casting experiments were carried out with the amount of PAA in the dispersion liquid varying from $1-6 \%$ wt. At $1 \%$ PAA concentration the green density at $\mathrm{pH} 10$ was higher than that at $\mathrm{pH} 6$, which is expected for the most stable suspension. For the $1 \%$ PAA concentration, the $\mathrm{pH}$ 10 suspension would expected to be more stable than the $\mathrm{pH} 6$ because of its higher repulsive forces, both electrostatic (-38 cf $-33 \mathrm{mV}$ ) and steric ("brush" cf "pancake" conformation). The highest green densities achieved with the $6 \%$ wt solutions were at $\mathrm{pH} 6$ (Figure 3), reversing the expected trend seen for the 1\% PAA solutions. The zeta potentials for these concentrated suspensions (6\% PAA) were evaluated using acoustophoresis (PenKem 7000) and gave similar values (Table 1) suggesting this reversal in trends is not associated with the electrostatic repulsion. Above PAA concentrations of around $2 \% \mathrm{wt}$, the green densities for the $\mathrm{pH} 6$ slurries are higher than those for the $\mathrm{pH} 10$ slurries. This could perhaps be attributed to the continued adsorption of PAA at pH 6 (Figure 2(b)) leading to a mixture of both "pancake" and "brush" 
conformations (Figure 2(d)). To support these hypotheses interparticle interaction potential calculations have been performed.

Interparticle interaction potentials have been calculated using a similar approach to that given in detail by Bergstrom et al [11], the results are shown in Figure 4. These gamma alumina particles are agglomerates with median volume diameters around $860 \mathrm{~nm}$. The primary particle sizes are around $20 \mathrm{~nm}$ and the porosity in the agglomerates was estimated to be around $75 \%$ from nitrogen adsorption-desorption isotherms. A reduced Hamaker constant was used by taking into account the particle pore volume by substituting the Hamaker constant for water for the one of alpha alumina. The adsorbed layer thickness has been estimated from the approximated molecular dimensions for a PAA molecule of 2000 molecular weight and from AFM measurements carried out using similar molecules $[8,12]$. Where to situate the charge plane, for interparticle potential calculations for an adsorbed polyelectrolyte layer is a much discussed problem [13]. We have chosen to take the plane as being at half the adsorbed layer thickness similar to the approach taken by Lewis et al for concentrated cement suspensions.[14]

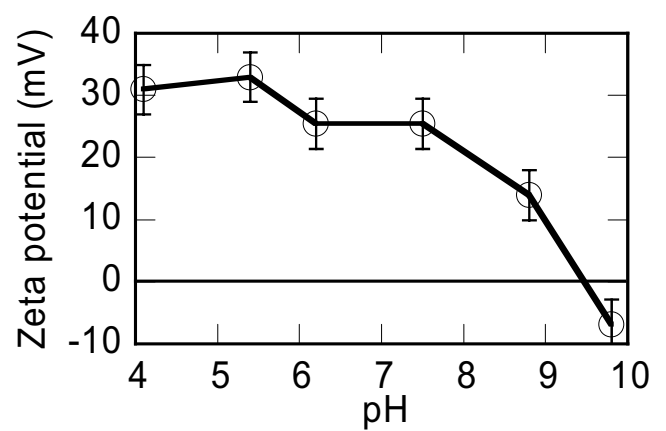

(a)

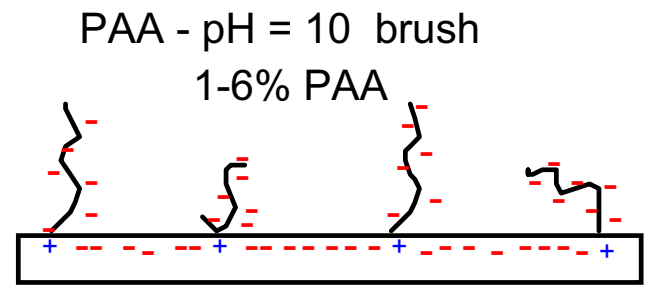

(c)

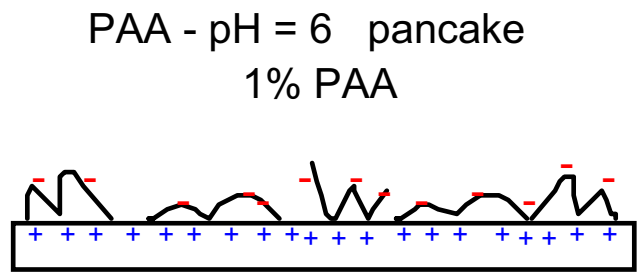

(b)

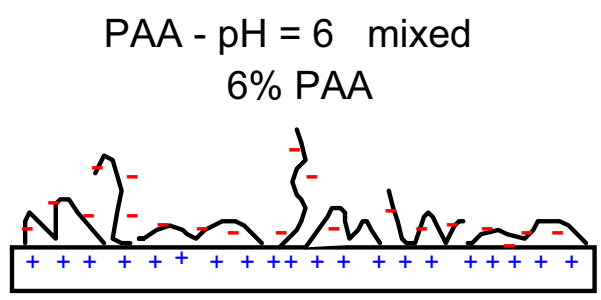

(d)

Figure 2. (a)Zeta potential as a function of pH for gamma alumina $\left(\mathrm{KNO}_{3} 0.01 \mathrm{M}\right)$ and schematic representations of PAA adsorption at (b) 1\%wt PAA, pH 6, (c) 1-6\%wt PAA, pH 10, (d) 6\% wt PAA pH 6. 


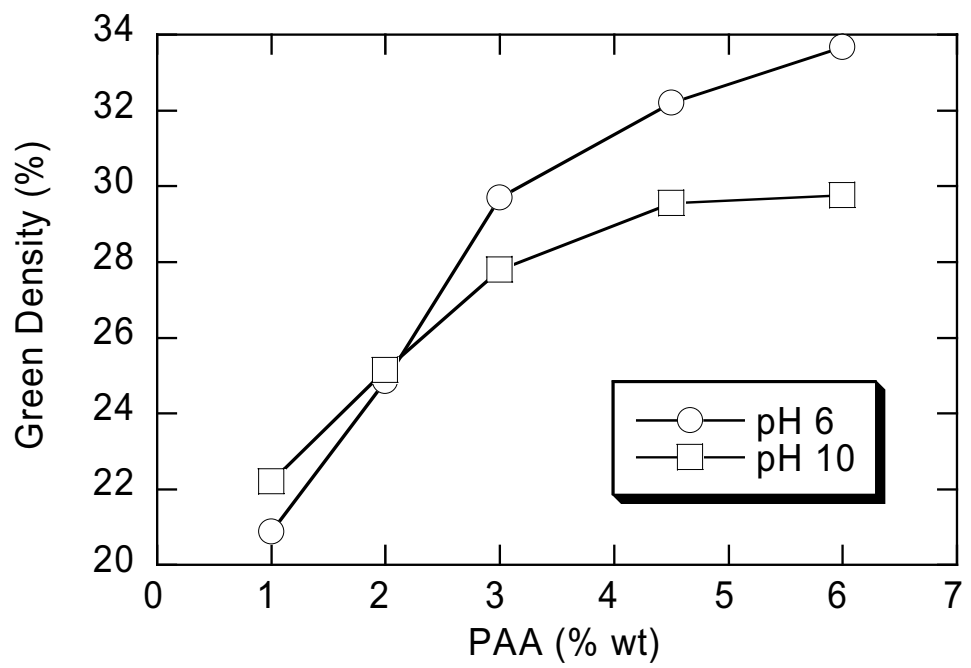

Figure 3. Density of gamma alumina green bodies after slip casting with PAA at pH 6 or 10 (lines are just guides for the eye).

Table 2. Zeta potential, ionic concentrations of the solutions and adsorbed polymer layer parameters used for the interparticle potential calculations.

\begin{tabular}{|c|c|c|c|c|}
\hline & $\begin{array}{l}1 \% \text { PAA, } \\
\text { pH } 6\end{array}$ & $\begin{array}{l}1 \% \text { PAA } \\
\text { pH } 10\end{array}$ & $\begin{array}{c}6 \% \text { PAA, } \\
\text { pH } 6\end{array}$ & $\begin{array}{l}6 \% \text { PAA } \\
\text { pH } 10\end{array}$ \\
\hline Zeta potential (mV) & -33 & -38 & -44 & -45 \\
\hline Ionic concentration $(\mathrm{M})$ & 0.2 & 0.26 & 1.53 & 2.0 \\
\hline $\begin{array}{l}\text { Adsorbed polymer chain - } \\
\text { volume fraction }\end{array}$ & 0.7 & 0.07 & 0.85 & 0.07 \\
\hline Adsorbed layer thickness (nm) & 3 & 5 & 5 & 5 \\
\hline $\begin{array}{l}\text { Distance of charge plane from } \\
\text { surface }(\mathrm{nm})\end{array}$ & 1.5 & 2.5 & 2.5 & 2.5 \\
\hline
\end{tabular}

The results for alumina powder dispersion in $\mathrm{HNO}_{3}(0.16 \mathrm{M})$ shows a primary minimum of around $30 \mathrm{kT}$. With PAA at $1 \% \mathrm{wt}$ as a dispersant solution, the primary minimum is reduced to about $8 \mathrm{kT}$ for $\mathrm{pH} 6$ and $4 \mathrm{kT}$ for the $\mathrm{pH} 10$ solutions, respectively (Figure 4(a)). This illustrates how the steric barrier reduces the magnitude of the attractive potential. The slightly smaller primary minimum for the $\mathrm{pH} 10$ solution indicates a slightly better stability, in agreement with the higher green density found under these conditions (Figure 3). For the $6 \%$ PAA solutions the situation is reversed with a more important steric effect for the $\mathrm{pH}=6$ dispersion when we assume 
the mixed "brush" and "pancake" conformation of the adsorbed PAA. Again these results agree qualitatively with the green densities as discussed above. The small differences between the interaction potentials as calculated for the $6 \%$ PAA solutions would not suggest such a large difference in the green densities. The assumptions made with respect to the adsorbed layer thickness (conformation), position of charge plane and volume fraction of adsorbed chains should be investigated in more detail. Techniques such as atomic force microscopy and molecular modeling of the alumina-PAA-water interface should help to further clarify the stabilizing mechanism.

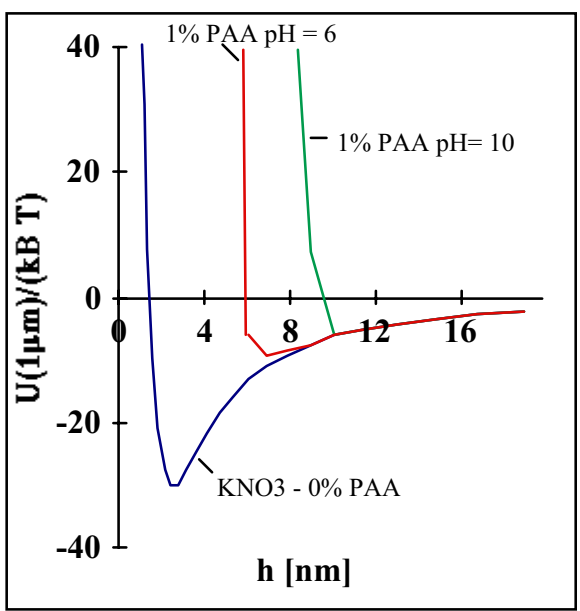

(a)

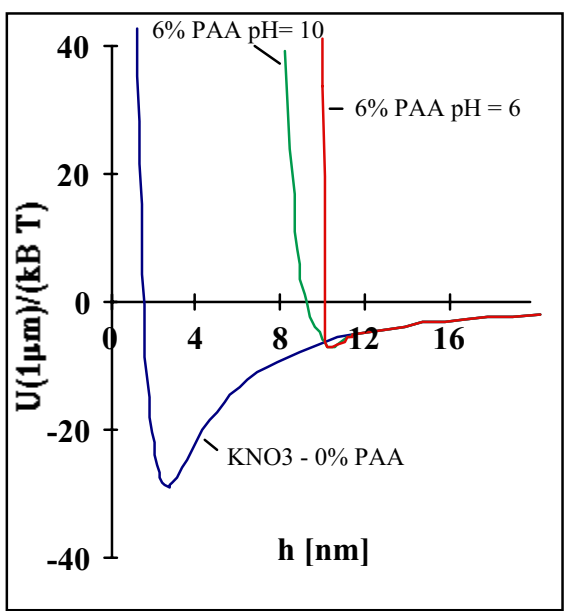

(b)

Figure 4. Interparticle interaction potentials (Table 1) for gamma alumina particles (diameter $0.86 \mu \mathrm{m}$ ).

\section{Attrition milling and slip casting}

The green densities achieved even with the best of the above dispersions $-6 \%$ wt PAA at pH 6 was still only $33 \%$. This is because the powders are heavily agglomerated and significant interagglomerate pores as well as the intra-agglomerate pores contribute to the overall porosity [4]. The green density can be improved significantly to around $45-50 \%$ by attrition milling with zirconia media [15]. The attrition milling reduces the median volume particle size, $\mathrm{d}_{\mathrm{v} 50}$ from about $700 \mathrm{~nm}$ to below $100 \mathrm{~nm}$ after $3 \mathrm{hrs}$ of milling (Figure 5 and Table 1)[16]. This is however, at the cost of contaminating our high purity starting powders with up to $2 \% \mathrm{wt}$ of zirconia from the milling media. All slip casting, both with and without milling, for the sintering experiments was made using the best dispersion conditions found above, i.e. $6 \% \mathrm{wt} \mathrm{PAA}$ solutions at $\mathrm{pH} 6$.

\section{Sintering}

The green and sintered densities are presented in Table 3. Milling improves significantly both the green and sintered densities for all powders studied. The green densities after milling approach $50 \%$, increasing from the very low 30 to $35 \%$ before milling; this is due to the significant de- 
agglomeration of the powders during the milling step. Without the milling step, the unseeded samples have very low sintered densities around $60 \%$. The alpha seeded sample shows a much improved density but is still low at around $86 \%$. All samples have densities $>97 \%$ after they have undergone $3 \mathrm{hrs}$ attrition milling. The milling significantly enhances densification in the alpha phase as well as reducing the gamma to alpha transition temperature for the pure and $\mathrm{Mg}$ doped gamma alumina. The seeded gamma aluminas show no change in the transformation temperature or the alpha sintering temperature within experimental error.

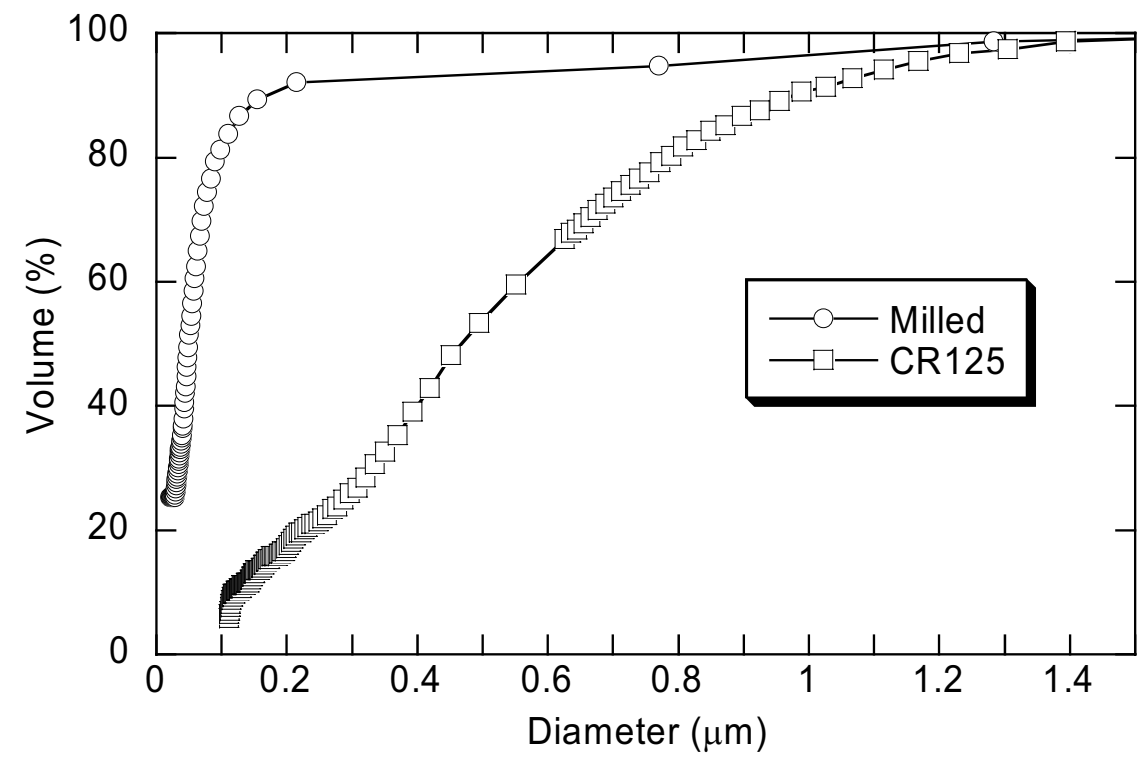

Figure 5. Cumulative volume particle size distributions of a typical gamma alumina powder used in the current study before ( $\square$ ) and (O)after 3 hrs attrition milling.

The above results were confirmed with dilatometry experiments on all of the samples described in Table 3. This suggests that the milling has a similar effect to the $9.4 \%$ of alpha alumina seeds on the gamma to alpha transformation temperature and sintering. That is to say, milling seems to give a similar number of nucleation sites as does the seeding with a sub-micron alpha alumina during the powder synthesis. An example of the dilatometry results for the Mg doped powder both before and after attrition milling, at a heating rate of $1 \% \mathrm{~min}$ is shown in Figure 6 . This shows the significant decrease in temperature associated with the milling step for both the first densification rate peak (associated with the transition from gamma to alpha alumina) and the second peak associated with sintering in the alpha phase. Typical microstructures are shown in Figure 7. The milled, gamma alumina and alpha seeded, powders give similar microstructures with elongated grains $>1 \mu \mathrm{m}$ at similar densities for the simple single step sintering cycle at $10^{\circ} /$ min to $1600^{\circ} \mathrm{C}$ (Figure 7 (a) and (b)). The Mg doped powder (milled and slip cast) with the same single step sintering cycle shows a similar grain size $>1 \mu \mathrm{m}$ but much less evidence for elongated grains. The same $\mathrm{Mg}$ doped powder (milled and slip cast) but with a two step sintering cycle $\left(10^{\circ} / \mathrm{min}\right.$ to $1390^{\circ} \mathrm{C}$, then $20 \mathrm{hrs}$ at $\left.1340^{\circ} \mathrm{C}\right)$ shows a much finer average grain size around $580 \mathrm{~nm}$. This slower densification route at lower temperatures therefore shows the advantage of less grain growth for a similar sample density. These grain sizes are still an order of magnitude greater than the starting gamma alumina grain size of $20 \mathrm{~nm}$. 
Table 3. Green density, sintered densities and apparent temperature of gamma to alpha transformation.

\begin{tabular}{lccccl}
\hline Sample & $\begin{array}{c}\text { Green } \\
\text { density } \\
(\%)^{*}\end{array}$ & $\begin{array}{c}\text { Sintered } \\
\text { density } \\
(\%)^{*}\end{array}$ & $\begin{array}{c}\text { Transformation } \\
\text { temperature }^{\#} \\
\left({ }^{\circ} \mathrm{C} \pm 5\right)\end{array}$ & $\begin{array}{c}\text { Sintered } \\
\text { grain size } \\
(\mu \mathrm{m})^{£}\end{array}$ & Grain shape \\
\hline CR 125 & 36.3 & 60.9 & 1235 & 0.5 & Vermicular \\
Milled (PAA) & 48.9 & 98.1 & 1200 & $>1$ & elongated \\
Mg Doped (Mg/Al 6799ppm) & 34.6 & 62.9 & 1240 & 1.0 & vermicular \\
Mg Doped - milled & 49.1 & 98.6 & 1185 & $>1$ & regular \\
Mg Doped - milled (2 step) ${ }^{\S}$ & 49.1 & $98.1^{\S}$ & - & $0.58^{\S}$ & $\begin{array}{l}\text { regular } \\
\text { octahedral }\end{array}$ \\
Seeded (9.4\% wt alpha) & 32.5 & 85.9 & 1195 & 1.0 & $\begin{array}{l}\text { regular } \\
\text { octahedral }\end{array}$ \\
Seeded (9.4\% wt alpha) - milled & 49.5 & 97.0 & 1205 & 1.0 & elongated \\
\hline
\end{tabular}

*corrected for $\mathrm{ZrO}_{2}$ contamination from the milling (measured using X-ray fluorescence spectroscopy) ${ }^{\text {\& }}$ sintered in single step at $1600^{\circ} \mathrm{C}$ except ${ }^{\S}$ two steps $1390-1320^{\circ} \mathrm{C}-20 \mathrm{hrs}$ ). ${ }^{\#}$ Deduced from differential thermal analysis (peak temperature, $10 \% \mathrm{~min}){ }^{\mathfrak{E}}$ Sintered grain size estimated from fracture surfaces except ${ }^{\$}$ Estimated from polished and etched surfaces counting around 500 grains.

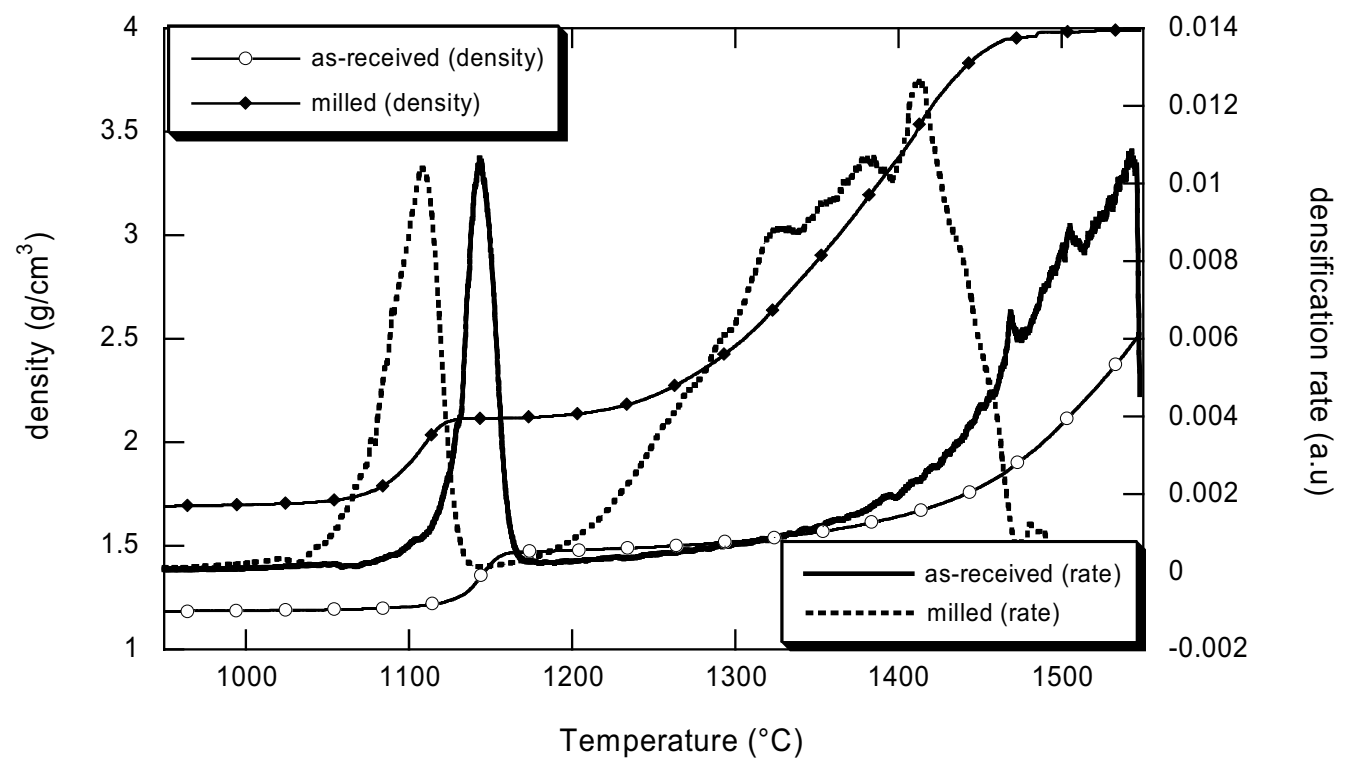

Figure 6. Dilatometry results showing density variations and densification rate curves for the $\mathrm{Mg}$ doped gamma alumina (slip cast samples from the as-received powder and after 3 hrs milling). 
To further improve upon this result it is felt that several aspects can be investigated, in particular; further reducing the state of agglomeration of the gamma alumina powders, the use of finer alpha alumina seeds, investigation of the development of the microstructures during the transformation step and reduction of the zirconia impurities. The state of agglomeration of the powders has been significantly improved by attrition milling but $10 \%$ of the particles (in volume) are still above $100 \mathrm{~nm}$ - this will still affect the green body homogeneity. Perhaps smaller milling media can help bring us towards a particle size distribution with all particles below $100 \mathrm{~nm}$ giving us the narrow size distribution known to help reduce final grain sizes in alpha alumina ceramics [17]. The use of a finer alpha alumina as a seed source should enhance the effects of densification and hopefully reduce the grain size if the "one seed leading to one grain" approximation can be assumed, similar to the early work on boehmites [18]. Zirconia impurities from milling have also been shown to have an adverse effect on alpha alumina sintering [17, 19]. Reduction of this effect by using suitable alumina milling media should also help us to reach a better control of the sintering process.

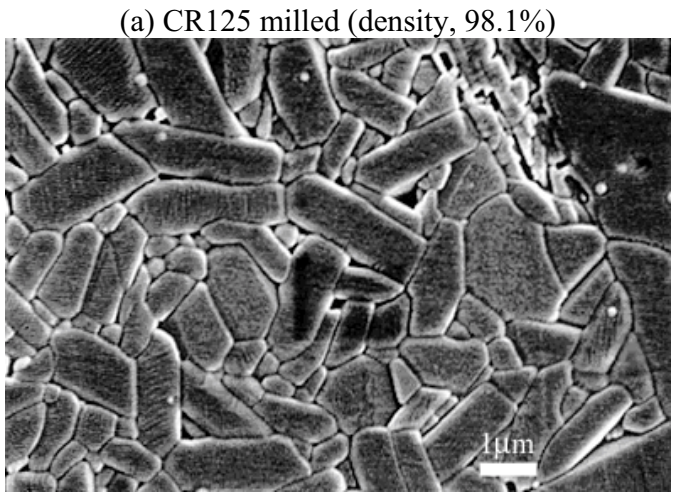

(c) Mg doped attrition milled, single step sintering, (density, 98.6\%)

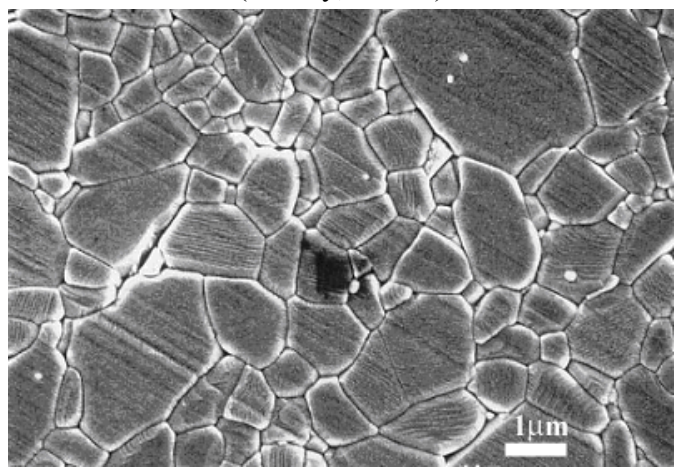

(b) $9.4 \%$ alpha seeded milled (density, $97.0 \%$ )

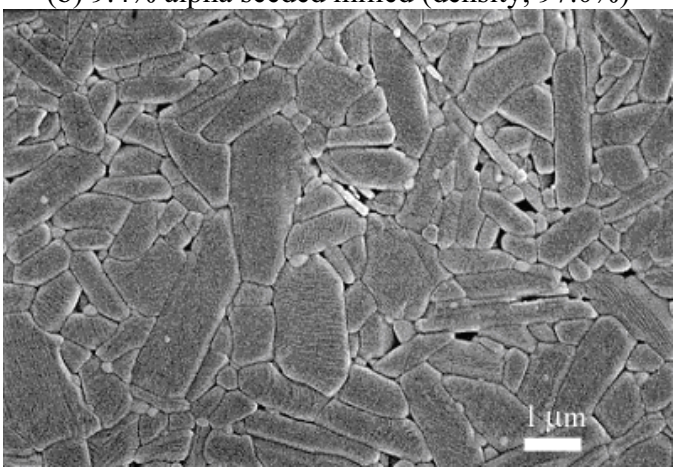

(d)Mg doped attrition milled, two step sintering, (density, 98.1\%)

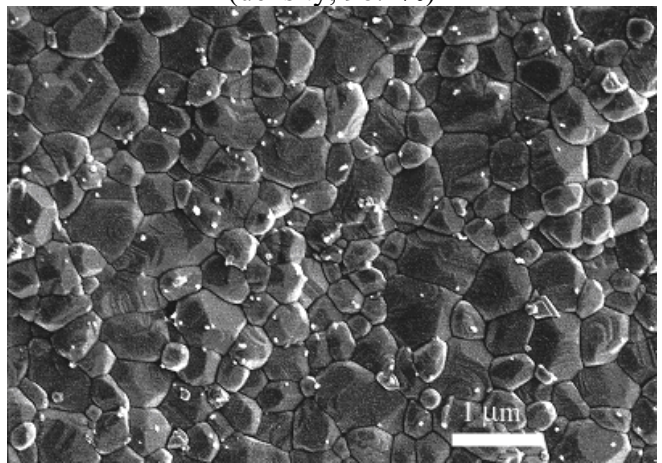

Figure 7. SEM micrographs of polished and thermally etched surfaces of the different sintered aluminas after attrition milling and slip casting (a) CR125 attrition milled (single step $\left.1600^{\circ} \mathrm{C}\right)$ (b) $\mathrm{Mg} 9.4 \%$ alpha seeded - attrition milled (single step $\left.-1600^{\circ} \mathrm{C}\right)(\mathrm{c}) \mathrm{Mg}$ doped attrition milled (single step $-1600^{\circ} \mathrm{C}$ ) (d) $\mathrm{Mg}$ doped attrition milled (two step sintering 1390-1320 ${ }^{\circ} \mathrm{C}$ $20 \mathrm{hr}$, white spots are zirconia impurities from milling) 


\section{CONCLUSIONS}

Colloidal processing of nanosized gamma alumina powders has been investigated. It was found that at $\mathrm{pH} \mathrm{6,} \mathrm{where} \mathrm{the} \mathrm{alumina} \mathrm{surface} \mathrm{is} \mathrm{positive,} \mathrm{dispersion} \mathrm{and} \mathrm{green} \mathrm{body} \mathrm{density} \mathrm{improves}$ as the concentration of the polyacrylic acid (PAA) is increased. For dispersions at $\mathrm{pH} 10$ the effect, was less marked. The differences have been attributed to the adsorption conformation of the PAA supported by interparticle interaction potential calculations. The proposed conformations were; brush-like at $\mathrm{pH} 10$ (all concentrations), pancake-like at $\mathrm{pH} 6$ (low PAA concentrations) and a mixture of brush and pancake conformations at $\mathrm{pH} 6$ (high PAA concentrations).

The best slip cast green bodies of the as-received gamma alumina gave relatively poor sintered densities of around $60 \%$. The powder with around $10 \%$ of alpha alumina seeds showed a significant improvement (density around $86 \%$ ). Such a result was mainly attributed to the state of agglomeration of the powders. All powders gave significantly higher green and sintered densities after $3 \mathrm{hrs}$ attrition milling, which reduced particle sizes by an order of magnitude from around $500 \mathrm{~nm}$ to $50 \mathrm{~nm}$. The gamma alumina samples after attrition milling and seeding with alpha alumina gave sintered densities over $98 \%$ but grain sizes still reach a micron. Magnesium doped gamma aluminas after attrition milling and slip casting gave similar grain sizes but without the significant elongation of the grains observed without doping. Sintering the doped gamma alumina (milled and slip cast) using a two stage sintering profile, rather than the simple heat and hold profile gave significantly finer grain sizes around $600 \mathrm{~nm}$.

Further work on aspects such as powder agglomeration, milling impurities, alpha alumina seed size and the sintering cycle should help us towards the fully dense alumina ceramics with sub $100 \mathrm{~nm}$ grain sizes.

\section{ACKNOWLEDGMENTS}

The authors would like to thank Calros Morais for the adsorption isotherms, Olivier Charvin for slip casting and Baikowski for powders.

\section{REFERENCES}

[1] J. H. Fendler (Ed), Nanoparticles and Nanstructured Films, Wiley VCHverlag, 1998, Weinheim.

[2] J. R. Groza and R.J. Dowding, Nanoparticulate Materials Densification, NanoStr. Mater., 7(7) (1996) 749-68.

[3] J. M. McHale, A. Auroux, A. J. Perrotta and A. Navrotsky, Surface Energies and Thermodynamic Phase Stability in Nanocrystalline Aluminas, Science, 277 (1997) 788-791.

[4] P. Bowen, C. Carry, From powders to sintered pieces: forming, transformations and sintering of nanostructured ceramic oxides, Powder Technology, 128 (2002) 248-255.

[5] P.-L Chang, F.-S. Yen, K.-C. Cheng and H.-L. Wen, Examinations on the critical and primary crystallite sizes during $t$ to a phase transformation of ultrafine Alumina Powders, Nanoletters, 1(5) (2001) 253-261.

[6] F. F. Lange, Powder Processing Science \& Technology for Increased Reliabilty, J.Am.Ceram.Soc, 72(1) (1989) 3-15.

[7] J. Cesarano III. I.A. Aksay, Stability of Aqueous alpha-A12O3 Suspensions with Poly(methacrylic acid) Polyelectrolyte, J.Am.Ceram.Soc., 71(4) (1988) 250-255.

[8] P. G. de Gennes, Polymers at an interface; a simplified view, Advances in Colloid and Interfaces Science, 27 (1987) 189-209. 
[9] H. G.Pederson, L. Bergström, Forces Measured between Zirconia Surfaces in Poly (acrylic acid ) Solutions J. Am. Ceram. Soc., 82 (4) (1999) 1-9.

[10] I. W. Chen \& X. H. Wang, Sintering dense nanocrystalline ceramics without final-stage grain growth, Nature, 404 (2000) 168-171.

[11] L. Bergström, C. H. Schilling, I. A. Aksay, Consolidation Behavior of Flocculated Alumina Suspension, J. Am. Ceram. Soc., 75 (12) (1992) 3305-3314.

[12] H. Kamiya, Y. Fukuda, Y. Suzuki, M. Tsukada, Effect of Polymer Dispersant Strucure on Electrosteric Interaction and Dense Alumina Suspension Behavior, J. Am. Ceram. Soc., 82 (12) (1999) 3407-3412.

[13] R. J. Flatt, Polymeric Dispersants in Concrete, Polymers in Particulate Systems: Properties and Applications, (Hackley V.A., Somasundaran P., Lewis J.A., Eds), Marcel Dekker, New York, 2001, pp. 247-294.

[14] J. A. Lewis, H . Matsuyama, G. Kirby, S. Morissette, J. F. Young, Polyelectrolyte effects on the rheological properties of concentrated cement suspensions, J. Am. Ceram. Soc., 83 (8) (2000) 1905-1913.

[15] P. Bowen, M. Staiger and H. Hofmann, C. Carry, Processing effects on the sintering behaviour of transition alumina powders, in, Sintering Science and technologies, eds, R.M. German, G.L. Messing, R.G. Cornwall The Pennsylvania State University, State College PA, USA, (2000) 171-176.

[16] M. Staiger, P. Bowen, J. Ketterer and J. Bohonek "Particle Size Distribution Measurement and Assessment of Agglomeration of Commercial Nanosized Ceramic Particles”, J. Dispersion Science and Technology, 23(5) (2002) 619-630.

[17] F-S. Shiau, T-T. Fang, T-H Leu, Materials Chemistry and Physics, 57 (1998) 33-40.

[18] G. L. Messing, M. Kumagai, Low-Temperature sintering of $\alpha$-alumina seeded boehmite gels, Am. Ceram. Soc. Bull., 73 (10) (1994) 88-91.

[19] A. Krell ,P. Blank, H. Ma, T. Hutzler, M. Nebelung, Processing of High-Density Submicrometer Al2O3 for new Applications, J. Am. Ceram. Soc., 86[4] (2003) 546-553. 